\title{
Sudden Cardiac Death and Post Cardiac Arrest Syndrome. An Overview
}

\author{
Endre Zima* \\ Semmelweis University, Heart and Vascular Center, Budapest, Hungary
}

\begin{abstract}
A satisfactory neurologic outcome is the key factor for survival in patients with sudden cardiac death (SCD), however this is highly dependent on the haemodynamic status. Short term cardiopulmonary resuscitation and regained consciousness on the return of spontaneous circulation (ROSC) is indicative of a better prognosis. The evaluation and treatment of SCD triggering factors and of underlying acute and chronic diseases will facilitate prevention and lower the risk of cardiac arrest. Long term CPR and a prolonged unconscious status after ROSC, in the Intensive Care Units or Coronary Care Units, indicates the need for specific treatment and supportive therapy including efforts to prevent hyperthermia. The prognosis of these patients is unpredictable within the first seventy two hours, due to unknown responses to therapeutic management and the lack of specific prognostic factors. Patients in these circumstances require the highest level of intensive care and aetiology driven treatment without any delay, independently of their coma state. Current guidelines sugest the use of multiple procedures in arriving at a diagnosis and prognosis of these critical cases.
\end{abstract}

Keywords: cardiac arrest, therapeutic hypothermia, cardiopulmonary resuscitation

Received: 10 July 2015 / Accepted: 28 September 2015

\section{Definition of Sudden CARDiac DeATH, INCIDENCE AND TREATMENT}

Based on data given in Morbidity and Mortality Weekly Report, sudden cardiac death (SCD) is responsible for $63 \%$ of deaths of cardiac origin in the US [1].

The incidence of SCD is 450.000 per year in the US, 400.000 per year in Western European countries and 3.000.000 per year worldwide. After admission to a hospital, and on the resumption of spontaneous circulation (ROSC), the survival rate is very poor, being in the range of about $5 \%$ in the US, between $5 \%$ and $7.9 \%$ in the EU, and below $1 \%$ worldwide $[2,3]$.

A large number of deaths result as a consequence of permanent severe neurological damage due to prolonged "no-flow anaesthesia" and "low-flow anaesthesia" from SCD to ROSC associated with complex resuscitation procedures [4].

The Seattle-study reported on over 12,000 SCD patients over a period of twenty four years, who had been treated by emergency medical services (EMS). An improvement in survival rates from $15.7 \%$, during the period between 1998 and 2001, to $17.5 \%$, beteween the period betweeen 1977 and 1981 was recorded. The long term outcome among patients who survived until discharged from hospital, also improved during this period $[5,6]$.

The analysis of data obtained from a nationwide cohort of 547.153 Japanese patients who presented with SCD between 2005 and 2009, showed that survival with a concomitant favourable neurologic status improved nearly two-fold. The survival to hospital discharge improved from $1.6 \%$ to $2.8 \%$ among all out-of hospital cardic arrest (OHCA) patients, from 2.1 to $4.3 \%$ in confirmed SCD, and from $9.8 \%$ to $20.6 \%$ among confirmed SCA with initial ventricular fibrillation (VF) [7].

The Canada registry, pooling the data of a population of more than 34.000 patients who presented with OHCA between 2002 and 2011, showed a significant improvement from $7.7 \%$ in 2002 to $11.8 \%$, in one-year survival rates [8].

The CARES registry, included 70.027 OHCA US patients, prospectively enrolled from 2000 to 2012, showed that survival to hospital-discharge improved significantly from $5.7 \%$ to $8.3 \%$, with an accompanying significant improvement in neurological function [9]. 
The main causes of SCD are lethal ventricular arrhythmias. Eighty three percent were either ventricular tachycardia (VT) or ventricular fibrillation (VF), of which $62 \%$ were recorded as VT and $8 \%$ as VF. In patients with cardiac arrest, the key elements in the "chain of survival" are early recognition of SCD, early "call for help" and the immediate start of high quality advanced life support, including chest compression, ventilation, early defibrillation and advanced post cardiac arrest (PCA) care. Treatment options for patients who have survived an episode of SCD, or who are at high risk of SCD, are antiarrhythmic drugs, implantable cardioverter-defibrillator (ICD) devices or both [10].

\section{ADVANCED CARE IN THE CHAIN OF SUR- VIVAL AND POST CARDIAC ARREST MAN- AGEMENT}

Post-cardiac arrest syndrome (PCAS) has been well documented over the last three decades in emergency, intensive care and acute cardiac care units. Cardiac arrest accompanied by "no-flow”, successive cardiopulmonary resuscitation $(\mathrm{CPR})$ and the concomitant "low-flow" haemodynamic state, results in a time- and CPR-quality-dependent reversible or irreversible hypoxic injury of organs. PCAS treatment starts immediately after ROSC, and is tailored to the physiologic needs of the patient's haemodynamic state. The aim of the therapeutic measures is to restore the normal cerebral function. The basics of the latter is a stable rhythm and an optimal cardiac output, in order to avoid neurological impairement and generalised ischaemic-reperfusion injury. Part of the systemic ischaemia reperfusion injury reaction causes a multiorgan pathophysiology, with cardiac, cardiovascular, neurologic, pulmonary, renal metabolic disorders, and a „sepsis-like” syndrome with specific complement activations. The survival limiting factor is the ischaemia tolerance and metabolic reserve of the brain. Ischaemia and ischaemia reperfusion injury, cause intense stress in the brain, including oxidative stress, microvascular injury, excitotoxicity, blood-brain barrier dysfunction and postischaemic inflammation initiated by neuronal, glial and endothelial cell death, or apoptosis $[11,12]$.

All the pathopysiologic disorders are dependent on the duration and quality of CPR. The main determinants of survival are the reversibility of post-cardiac arrest (PCA) brain injury, PCA myocardial dysfunc- tion and the aetiology and recurrency of SCD. All the factors involved in the genesis of cardiac arrhythmia need to be ruled out and treated. Such factors may include structural diseases, e.g. acute or chronic coronary artery disease, cardiomyopathies, trigger mechanisms such as abnormal automaticity, premature beats or modulating factors such as ischaemia, electrolyte-disorders, sympathicotonia, drug effects modulating repolarisation. One of the most important dignostic and therapeutic methods is urgent coronary angiography and revascularisation, since at least seventy percent of the SCD patients have been proven to have a significant coronary lesion [13].

Neurologic injury is the most common cause of death in OHCA patients. Preventing hyperthermia during the first few hours after CA reduces the risk of neurologic injury. Therapeutic hypothermia (TH), and since 2015, forced normothermia, are part of the advanced PCAS care intended to save brain and vital organs from ischaemic-reperfusion injury [14].

Randomised clinical trials have shown improved outcome using $\mathrm{TH}$. Those adults remaining comatose after CPR for OHCA, with initial rhythm of VF, a temeperature reduced within minutes to hours after ROSC to $32-34^{\circ} \mathrm{C}$ for twelve to twenty four hours, had a significantly better survival. [15]

The Hachimi-Idrissi trial reported improved lactate and $\mathrm{O}_{2}$ extraction in comatose adult patients who were cooled after ROSC [16]. Other studies showed a benefit after therapeutic hypothermia in comatose survivors of SCD caused by non-shockable rhythm [17].

Janata et al [2009] conducted a meta-analysis of thirty seven non-randomized trials and fifteen controlled studies and showed that the pooled absolute risk reduction gained with this new treatment modality is approximately twenty five percent, with a number needed to treat of 5 [18].

The 2010 ERC Guidelines did not agree with the opinion that all unconscious adult patients with spontaneous circulation after $\mathrm{VF}$ associated to OHCA should be cooled to $32-34^{\circ} \mathrm{C}$ starting as soon as possible and continuing for twelve to twenty four hours. However, 2015 guidelines accept normothermia as an option, suggesting that the patient's temperature be reduced to $32-36{ }^{\circ} \mathrm{C}$. It is suggested that the maintainance of normotermia or hypothermia be facilitated by sedatives and neuromuscular blocking agents to prevent shivering [19]. 
The technique of therapeutic hypothermia has three phases, the induction of cooling, maintenance and rewarming. External cooling is performed using traditional icepacks placed on the groin, axilla, and sides of neck together with surface temperature devices to monitor temperature changes. Internal cooling may be started by infusion of $30 \mathrm{mg} / \mathrm{kg}$ of $4^{\circ} \mathrm{C}$ saline. This is capable of decreasing core temperature by a maximum of $1.5^{\circ} \mathrm{C}$. Intravascular cooling enables more precise temperature control than external methods and endovascular heat-exchange catheters enable a fast induction and rewarming. However this is associated with a higher cost due to the expensive equipment which is required.

The basic intensive support of the comatose PCAS patient includes mechanical ventilation, sedation with short acting agents, e.g.propofol and opioids and neuromuscular blockade. Invasive haemodynamic monitoring, urine output, core temperature and neurologic function monitoring is strongly recommended. Supportive intensive care includes balanced $\mathrm{K}, \mathrm{Ca}$ and $\mathrm{Mg}$ electrolyte-supply, volume therapy based on volumetric monitoring, parenteral or enteral feeding, antibiotics based on microbiological cultures and prophylaxis of decubitus lesions.

\section{WHAT IS THE PROGNOSIS AFTER SUCCESS- FUL CPR AND PCAS TREATMENT?}

Unfortunately it is frequently difficult to arrive at an exact prognosis in a comatose PCAS patients. It has been suggested that after seventy two hours of treatment, the absence of cornea or pupil reflex and the presence of continuous convulsion and myoclonus, is indicative of a poor outcome. The recommendations of the European Resuscitation Council [2014 ] aimed to clearify all the prognostic factors and give exact perspectives that could help in the continuation of maximal intensive care or withdrawal of supportive therapy, based on the recognition of adverse neurologic events [20].

There are several limited but robust points for prognosis. During examination of neurologic function, account of the residual effects of sedatives, opioids and neuromuscular blocking agents must be taken ito account. It is important to appreciate that at ROSC, bilateral absence of pupillary light reflex has very limited value in predicting a poor outcome, while myoclonus within seventy two hours from ROSC is not consistently associated with a poor outcome. At seventy two hours from ROSC, a bilaterally absent pupillary light reflex predicts poor outcome independently of therapeutic hypothermia usage. Moreover, a bilaterally absent corneal reflex is slightly less specific than a pupillary reflex, in predicting a poor outcome. At the same time, an absent motor response or extensor response to pain and a Glascow Coma Scale of one to two, has a seventy four percent sensitivity in predicting a poor outcome as was a status myoclonus, starting within forty eight hours from ROSC. Additionally, the absence of EEG reactivity to external stimuli and the presence of status epilepticus in comatose PCAS patients at seventy two hours after ROSC, is indicative of a poor outcome. Bilateral absence of the N20 wave in short-latency somatosensory evoked potentials (SSEPs) predicts brain death or a vegetative status. Recommendations point to the useof these criteria only in combination with other predictors, since there is no standardised technique or hard evidence as the recommendations are based on few studies.

The availability of biomarkers is limited though they can be useful if combined with other prognostic factors, if used at 48-72 hours from ROSC and measured repeatedly for at least twenty four hours after ROSC. Imaging tecniques such as brain CT or MRI are useful to exclude the causes of coma or subarachnoid haemorrhage and may help to detect cerebral oedema and anoxic-ischaemic cerebral injury. Since the methodology is inconsistent, current recommendations indicate that the use of brain CT and MRI is made only in combination with other predictors [20].

\section{REFERENCES}

1. Centers for Disease Control and Prevention (CDC). Statespecific mortality from sudden cardiac death - United States, 1999, MMWR Morb Mortal Wkly Rep, 2002;51:123-6.

2. Myerburg RJ, Castellanos A. Cardiac arrest and sudden cardiac death. Braunwald E, ed. Heart Disease: A Textbook of Cardiovascular Medicine. 5th ed. Philadelphia, Pa: WB Saunders; 1997:742-779.

3. de Vreede-Swagemakers JJ, Gorgels AP, Dubois-Arbouw WI, et al. Out-of-hospital cardiac arrest in the 1990's: a populationbased study in the Maastricht area on incidence, characteristics and survival. J Am Coll Cardiol. 1997;15;30(6):1500-1505.

4. Lloyd-Jones D, Adams R, Carnethon M, et al. Heart Disease and stroke statistics- 2009 update. A report from the American Heart association Statistics Committee and Stroke Statistics Subcommittee. Circulation. 2009;119:480-6.

5. Rea TD, Eisenberg MS, Becker LJ, Murray JA, Hearne T. Temporal trends in sudden cardiac arrest: a 25-year emergency medical 
170 - The Journal of Critical Care Medicine 2015;1(4)

services perspective. Circulation. 2003;107:2780-2785.

6. Rea TD, Crouthamel M, Eisenberg MS, Becker LJ, Lima AR. Temporal patterns in long-term survival after resuscitation from out-of-hospital cardiac arrest. Circulation. 2003;108:11961201

7. Kitamura T1, Iwami T, Kawamura $\mathrm{T}$, et al. Nationwide improvements in survival from out-of-hospital cardiac arrest in Japan. Circulation. 2012;126(24):2834-43.

8. Wong MK, Morrison LJ, Qiu F, et al. Trends in Short- and LongTerm Survival among Out-of-Hospital Cardiac Arrest Patients Alive at Hospital Arrival. Circulation. 2014;130:1883-90.

9. Chan PS, McNally B, Tang F, et al. Recent Trends in Survival from Out-of-Hospital Cardiac Arrest in the United States. Circulation. 2014;130:1876-82.

10. Bayes de Luna A, Coumel P, Leclercq JF. Ambulatory sudden cardiac death: Mechanisms of production of fatal arrhythmia on the basis of data from 157 cases. Am Heart J. 1989;117:151-9.

11. Jenei ZM , Zima E , Csuka D, et al. Complement activation and its prognostic role in post-cardiac arrest patients. Scandinavian Journal of Immunology. 2014;79:404-9.

12. Jenei ZM , Szeplaki G, Merkely B , et al. Persistently elevated extracellular HSP70 (HSPA1A) level as an independent prognostic marker in post-cardiac-arrest patients. Cell Stress \& Chaperones. 2013;18: 447-54.
Available online at: www.jccm.ro

13. Spaulding CM, Joly LM, Rosenberg A, et al. Immediate coronary angiography in survivors of out-of-hospital cardiac arrest. N Engl J Med. 1997;336:1629-33.

14. Monsieurs KG, Nolan JP, Bossaert LL, et al. ERC Guidelines 2015 Writing Group. European Resuscitation Council Guidelines for Resuscitation 2015: Section 1. Executive summary. Resuscitation. 2015;95:1-80.

15. Bernard SA, Gray TW, Buist MD, et al. Treatment of Comatose Survivors of Out-of-Hospital Cardiac Arrest with Induced Hypothermia. N Engl J Med 2002;346:557-63.

16. Hachimi-Idrissi S, Corne L, Ebinger G, Michotte Y, Huyghens L. Mild hypothermia induced by a helmet device: a clinical feasibility study. Resuscitation. 2001;51:275-81.

17. Bernard SA, Jones BM, Horne MK. Clinical trial of induced hypothermia in comatose survivors of out-of-hospital cardiac arrest. Ann Emerg Med. 1997;30:146-53

18. Janata A, Holzer M. Hypothermia After Cardiac Arrest. Prog Cardiovasc Dis. 2009;52:168-179.

19. Nolan JP, Soar J, Zideman DA, et al. European Resuscitation Council Guidelines for Resuscitation 2010 Section 1. Executive summary. Resuscitation. 2010;81:1219-76.

20. Sandroni C. Prognostication in comatose survivors of cardiac arrest. Resuscitation. 2014;85:1779-1789. 